\title{
Fast and accurate face orientation measurement in low-resolution images on embedded hardware
}

\author{
Dries Hulens, Kristof Van Beeck and Toon Goedemé \\ EAVISE, KU Leuven, Sint-Katelijne-Waver, Belgium \\ \{dries.hulens, kristof.vanbeeck, toon.goedemé\}@kuleuven.be
}

\begin{abstract}
Keywords: Face angle estimation, gaze direction, pose estimation, embedded hardware
Abstract: In numerous applications it is important to collect information about the gaze orientation or head-angle of a person. Examples are measuring the alertness of a car driver to see if he is still awake, or the attentiveness of people crossing a street to see if they noticed the cars driving by. In our own application we want to apply cinematographic rules (e.g. the rule of thirds where a face should be positioned left or right in the frame depending on the gaze direction) on images taken from an Unmanned Aerial Vehicle (UAV). For this an accurate estimation of the angle of the head is needed. These applications should run on embedded hardware so that they can be easily attached to e.g. a car or a UAV. This implies that the head angle detection algorithm should run in real-time on minimal hardware. Therefore we developed an approach that runs in real-time on embedded hardware while achieving excellent accuracy. We demonstrated these approaches on both a publicly available face dataset and our own dataset recorded from a UAV.
\end{abstract}

\section{INTRODUCTION}

In this paper we propose an efficient methodology to perform accurate face orientation estimation. Nowadays such techniques prove to be essential for several applications, ranging from the detection of abnormal behavior in surveillance cameras, attentiveness measurements in e.g. dangerous traffic situations and so on. However, current existing systems are often infeasible to be employed in real-life applications. This is mainly due to the fact that they often employ either a wide range of models to perform accurate detection (e.g. a new model for a small step size in degrees) or their detection pipeline imposes severe constraints on the required hardware or image resolution (high image resolution is needed for the detection of facial features). The latter is true for the most recent state-ofthe-art algorithms which are based on Convolutional Neural Network (CNN) approaches (see Section 2). In this paper we aim to develop an approach that overcomes these limitations: our objective is an efficient face orientation estimation algorithm that is able to run with high accuracy on minimal hardware. Here we focus on estimating the pan angle, but the same method can be used for e.g. the tilt angle of the head. Our goal is to build an autonomous virtual cameraman (UAV with camera and processing power) where all processing is performed on an on-board embedded

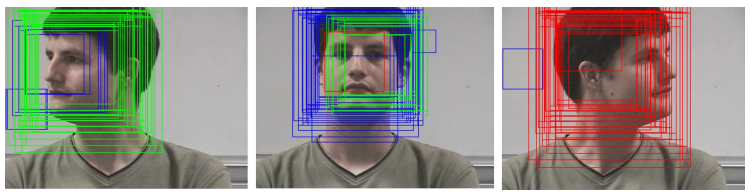

Figure 1: Image containing detected face candidates. Green: output from left model, blue: output from frontal model, red: output from right model.

hardware platform. An autonomous virtual cameraman films actors independently of a human operator, adjusting its position in order to comply with basic cinematographic rules, such as the rule of thirds. This rule signifies that a person looking to the right (this is most likely where the action is taking place) should be positioned on $1 / 3$ on the left of the frame (and vice versa) to leave some empty image space for the action. To apply this rule we need to detect the face orientation on the images taken by the UAV. Hereby the UAV can steer itself to position the person left or right in the frame depending on his face orientation like in (Hulens et al., 2014) where a pan-tilt unit is used to satisfy this rule. An even harder cinematographic rule is that the virtual cameraman should maintain a certain shot for a longer time e.g. keeping a skier speeding downhill in a profile close-up shot. This implies that the exact angle of the face should be detected so that the UAV can hold its position relatively to- 
wards the face. Evidently, due to the hardware constraints, our algorithm should be lightweight. For this, we propose a methodology, based on only three detection models, which are able to estimate the face pan angle with excellent accuracy and real-time performance whilst running on an embedded platform. For validation, we employed both a publicly available dataset and composed our own database captured from a UAV.

The remainder of this paper is structured as follows: In Section 2 we relate our method with the current literature in head pose estimation. In Section 3 we explain how our approach works. Section 4 shows our results and in Section 5 conclusions are drawn and future work is discussed.

\section{RELATED WORK}

Head pose estimation is a vital component for numerous applications like pedestrian safety (Schulz and Stiefelhagen, 2012; Rehder et al., 2014; Yano et al., 2014) where it is essential to know if the pedestrian anticipated possible danger (looking towards it) or for measuring car driver attentiveness (Paone et al., 2015; Tawari et al., 2014; Oyini Mbouna et al., 2013) to see if the driver is still alert. For those applications several methods are developed the last few years that are divided into two main categories: model-based and appearance-based approaches.

In the first approach the location of facial features (e.g. Haar features) combined with a geometrical face model is used to determine the head pose. The latter approach uses information of the entire facial region and a separate detector is trained for different poses. Examples of model-based approaches are (Pyun et al., 2014; Shbib et al., 2014) where they first detect the face and search for features that correspond to essential points within that region (e.g. mouth, nose and eyes). Depending on the spatial distribution of those features a pose estimation is accomplished. Another model-based approach is presented in (Liew and Yairi, 2015) where they overlay a 3D model of a human head with a 2D input image by connecting the facial features of the 2D images with features of the 3D model. Using these methods on-board a UAV would result in low accuracy, since it is difficult to find the aforementioned facial features in these low resolution images.

In appearance-based approaches generally a decision tree is learned where the leaves correspond with a face at a certain angle. An example is (Benfold and Reid, 2009), where they detect the gaze orientation of people to infer interesting areas or events using ran- domized ferns with decision branches based on both histogram of oriented gradients (HOG) and color features. In (Liu et al., 2014) and (Lu and Tan, 2013) a manifold learning method is used to estimate the head pose. They first extract features (e.g. intensity) from a training set of head images and perform a manifold analysis to learn a low-dimensional pose manifold where after the low-dimensional features are used to learn a multiple linear regression function. Prior techniques work well in practice. However these methods rely on an optimal head localization: if the localization is not optimal (e.g. the bounding box is not centered exactly on the head) a large deviation in head orientation occurs.

A totally different approach is to use 3D sensors and depth-images to estimate the pose of a face. In (Fanelli et al., 2011) they estimate the 3D coordinate of the nose tip using random regression forests trained on data captured from a range scanner while in (Marks and Jones, 2015) triangular patch features are extracted from the depth image of a persons' head and matched with a learned 3D model to estimate the head pose. As cited in other work of (Fanelli et al., 2012) the disadvantages of depth cameras are that the most accurate ones work with structured infrared light or time-of-flight which cannot be used outside. Other cameras like stereo-setups do work outside but produce very noisy reconstructions. In addition, it is not desirable to mount a stereo setup or depth sensor on a UAV due to their weight and power consumption as compared with a simple camera.

Our approach is also appearance-based but differs significantly from previous methods since it uses only three models (left-, right- and frontal-oriented) to estimate both the head pose and perform face detection simultaneously which accelerates the algorithm tremendously. There is no need for training a complicated decision tree and no time is spent on searching for additional facial features. Furthermore we use a simple light-weight webcam, to provide images, which can easily be mounted on a UAV or other embedded application.

\section{APPROACH}

The goal of our approach is to estimate the pan angle of a face in a new image. To achieve this we use three models of a face $\left(-90^{\circ},+90^{\circ}\right.$ and $\left.0^{\circ}\right)$ which are trained using the Viola and Jones framework (Viola and Jones, 2001) (further referred as V\&J). This framework is a strong binary classifier constructed out of several weak detectors. The weak detectors use simple features (Haar features) to decide if a certain 


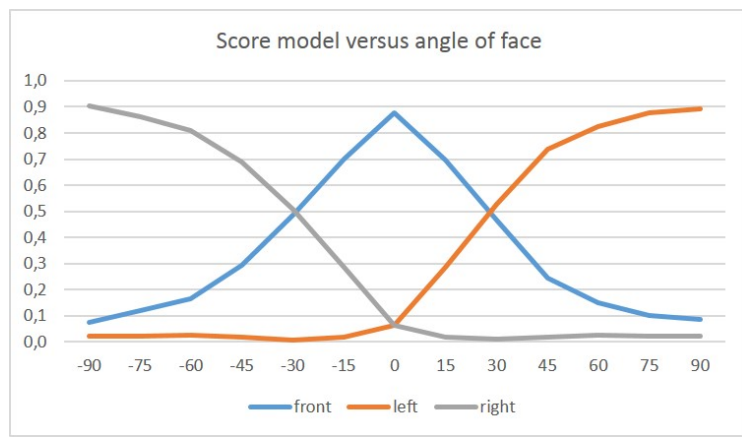

Figure 2: Relationship between the score of the model running over an image and the angle of the persons' face in the image. Orange: Left looking model, Blue: Frontal looking model and Gray: Right looking model

image patch is part of the face or not. The detectors are placed in a cascade and if the image patch passes through all of the cascade stages, it is classified as positive. In our case only two models are trained $\left(90^{\circ}\right.$ and $0^{\circ}$ ) and to evaluate $-90^{\circ}$ the image is mirrored and the $90^{\circ}$ model is used. For the sake of simplicity we talk about three models.

For each new input image, each of these three models thus returns a score indicating the probability of that image patch containing a face at that specific angle. The score they return is actually the number of detections without employing non maxima suppression as seen in figure 1. When the face is e.g. looking to the left, the left model will evidently output a higher probability score than both the right and frontal model.

We employed the face dataset of (Gourier et al., 2004) to examine the relationship between the scores of the V\&J models and the angle of the person. The face dataset contains 2790 monocular face images of 15 persons with variations of pan and tilt ranging from $-90^{\circ}$ to $+90^{\circ}$ in steps of $15^{\circ}$. We used 520 of the 780 images with a tilt angle of $0^{\circ}$ (260 images +260 mirrored images) as training data. For each of these thirteen angles we evaluated our three V\&J models. These scores are then normalized w.r.t. each other such that their sum is 1 . Because the scores are actually the number of detections they are normalized to be independent of the image quality or light conditions that can affect the number of detections. Figure 2 displays this normalized detection score for each of these angles. As seen a clear relationship exist. We propose two ways to exploit this to calculate the face angle in an image. In the next section we first exploit a naive linear model approach, called baseline approach. In section 3.2 we present our new more accurate approach, coined Angle Model Approach.

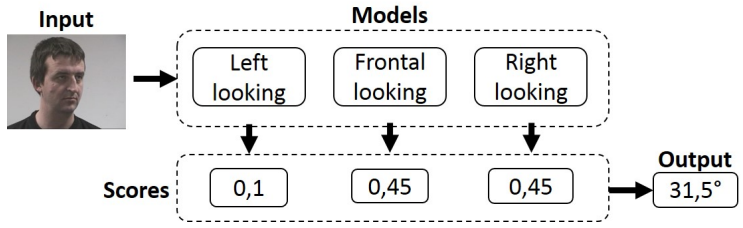

Figure 3: Our three V\&J models are validated on a new input image and output a score for each model. An angle is linear interpolated out of these scores.

\subsection{Baseline Approach}

Since the individual detection scores of the three models are normalized according to the method given above and assuming to have a linear relationship between the detection score and the angle of the face, we can derive the angle of a new input face image as a simple weighted sum of the detection scores. This is formulated as:

$$
\alpha=S_{\text {left }} \cdot\left(-90^{\circ}\right)+S_{\text {front }} \cdot\left(0^{\circ}\right)+S_{\text {right }} \cdot(9
$$

Where $\alpha$ is the angle estimation of the new input face image and $S_{\text {left }}, S_{\text {front }}$ and $S_{\text {right }}$ are respectively the score of the left, front and right model. The middle term is added to the formula for the sake of clarity, but is of course neglected due to the multiplication with $0^{\circ}$. Since the scores are normalized such that $\sum S_{i}=1, S_{\text {front }}$ is not ignored but is incorporated in $S_{\text {left }}$ and $S_{\text {right }}$. An example of this approach is seen in figure 3.

We evaluated this baseline approach on the (Gourier et al., 2004) dataset. Figure 4 gives accuracy results. When e.g. an error of $\pm 15^{\circ}$ is allowed, $64 \%$ of the samples are correctly classified with this simple approach. This approach runs at 13,34fps (frames per second) $(640 \times 480$ pixels $)$ on a desktop computer containing an Intel i7 processor and 20GB memory. The input image can be much smaller (until the face is undetectable), resulting in a significant acceleration.

\subsection{Angle Model Approach}

As we closely examine figure 2 the relationship between the model scores and the angles are not completely linear. Hence we propose that a model (the Angle Model) of the scores for every of the 13 angles (from $-90^{\circ}$ to $90^{\circ}$ in steps of $15^{\circ}$ ) is learned and the scores of a new input image are compared with the model.

\subsubsection{Construction of the Angle Model}

As previously mentioned, we ran our three V\&J models over a set of images with 13 different angles. 


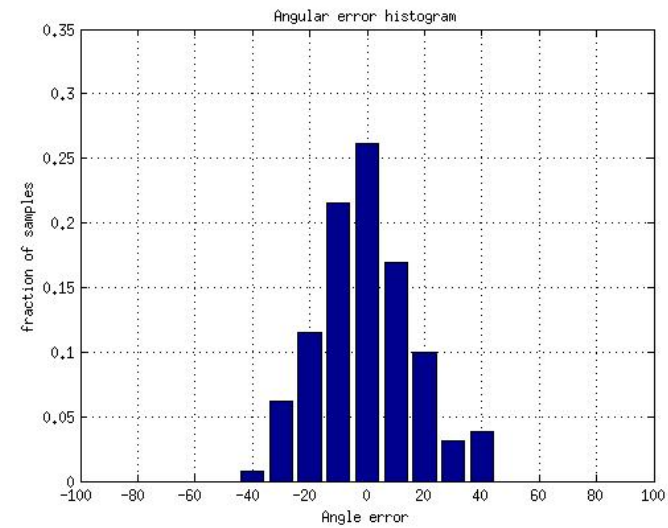

Figure 4: Accuracy results Baseline

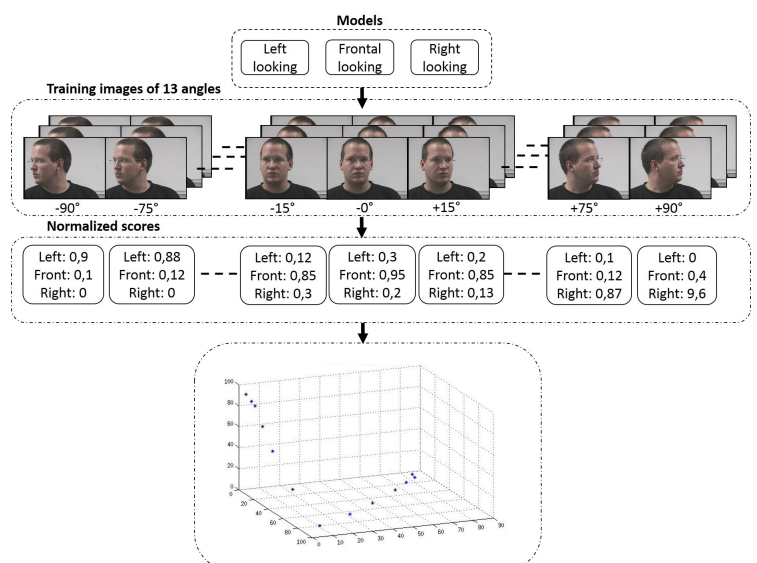

Figure 5: Overview of the training of the angle model

The scores of every angle-set are normalized and displayed in figure 2. In order to make our angle model we treated these normalized scores as a 3D point $p_{i}$ for every angle. Next each 3D point is plotted in a 3D map that forms the Angle Model. The workflow is seen in figure 5.

As an example: the left most point in the map has a left score of about $90 \%$ and a frontal and right score of about $5 \%$, this point corresponds evidently with a face looking fully to the left or an angle of $-90^{\circ}$. This angle model is trained on the desktop computer previously mentioned at a single core and took only 76 seconds to learn a model from 520 images (we divided the used dataset into a learning-part (520 images) and a test-part (260 images)).

\subsubsection{Detection and angle estimation}

The estimation of the face-angle in a new image takes place in a similar way as the training. For each new input image a face is detected using the three V\&J models. Each model outputs a score and the three val-

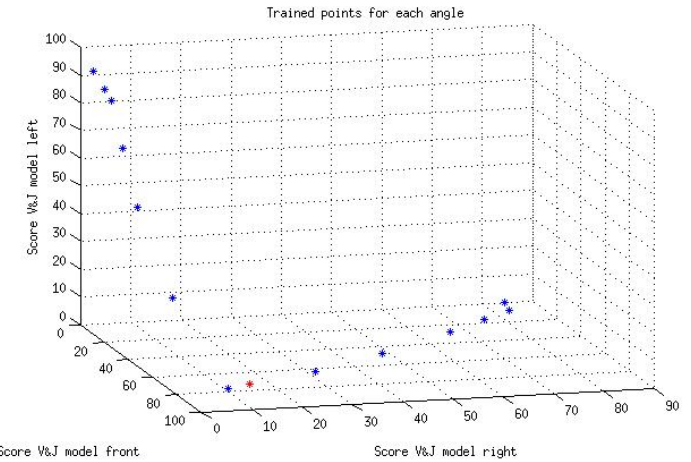

Figure 6: Angle model in blue. New input $p_{\text {new }}$ in red.

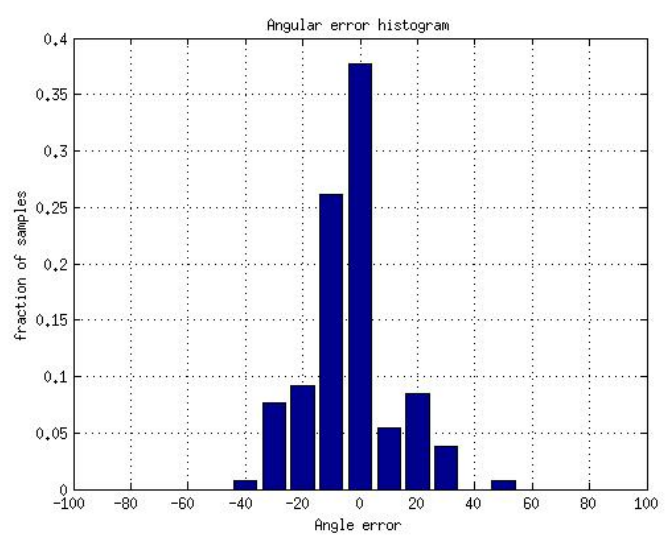

Figure 7: Accuracy results Angle Model

ues are normalized and combined in a new 3D point $p_{n e w}$. This new point is plotted in our angle model as in figure 6 . To calculate the angle $\alpha$ corresponding to $p_{\text {new }}$ the Euclidean distances $d_{1}$ and $d_{2}$ are calculated between $p_{\text {new }}$ and the two nearest points $p_{1}$ and $p_{2}$ in our angle model that correspond with $\alpha_{1}$ and $\alpha_{2}$. Since $d_{1}$ and $d_{2}$ are known, $\alpha$ can easily be interpolated with equation 2 .

$$
\alpha=\alpha_{1}+\frac{d_{1}}{d_{1}+d_{2}}\left(\alpha_{2}-\alpha_{1}\right)
$$

The accuracy results are seen in figure 7 . When e.g. an error of $\pm 15^{\circ}$ is allowed, $72 \%$ of the samples are correctly classified. This approach runs at 13,4fps on the previously described desktop which is about as fast as the Baseline Approach but with a noticeably better accuracy. If an error of only $\pm 5^{\circ}$ is allowed, we see an improvement of $12 \%$ more samples that is correctly classified as compared to the Baseline Approach (38\% vs. $26 \%$ correctly classified). 


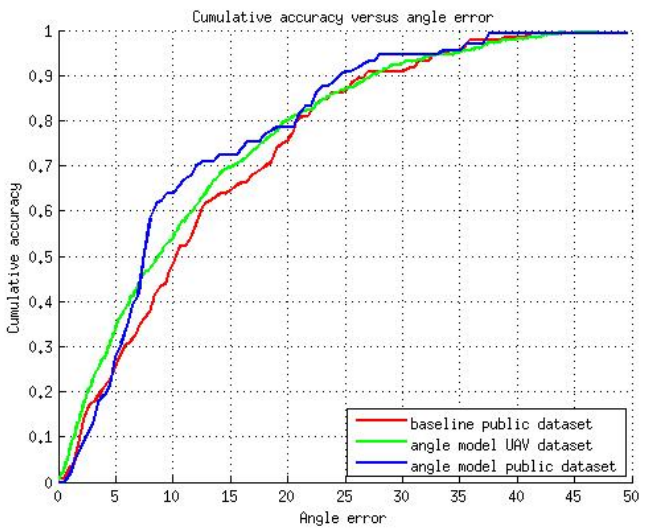

Figure 8: Accuracy results

\begin{tabular}{c|c|c} 
Method & range & $\begin{array}{c}\text { Mean } \\
\text { Absolute } \\
\text { Error }\end{array}$ \\
\hline Benfold \& Reid & $-180^{\circ} \ldots 180^{\circ}$ & $37,9^{\circ}$ \\
\hline Rehder & $-180^{\circ} \ldots 180^{\circ}$ & $19^{\circ}$ \\
\hline Ours-Baseline & $-90^{\circ} \ldots 90^{\circ}$ & $\mathbf{1 3}^{\circ}$ \\
\hline Ours-Model based & $-90^{\circ} \ldots 90^{\circ}$ & $\mathbf{1 1 , 2 5}^{\circ}$
\end{tabular}

Table 1: Mean absolute error for both approaches

\section{EXPERIMENTS AND RESULTS}

We conducted our experiments both on a publicly available dataset (Gourier et al., 2004) with given ground-truth and on our own dataset captured from a UAV where the compass of the UAV delivered the ground-truth. Since we want to run our algorithm onboard a UAV, experiments are performed on a desktop computer as well as on an embedded computer on the UAV itself. One of the experiments we performed was an accuracy measurement of both the baseline approach and the angle model approach. Results can be seen in figure 8 . We can see a slightly better performance of the angle model approach both on the dataset and the images taken by the UAV.

Results of the angle model approach can be seen in Figure 9.

Next the mean absolute error is calculated for both approaches as can be seen in Table 1 .

It is not trivial to compare our work with others because of the use of non-publicly available datasets, or a wider/smaller range of angles that can be detected. In (Benfold and Reid, 2008) they use randomized ferns to estimate the head angle between $-180^{\circ}$ and $180^{\circ}$ which yields in a mean absolute error of $37,9^{\circ}$. In (Rehder et al., 2014) a logic regression classifier is trained for four different orientations together with motion information from a KLT tracker. They reach a mean absolute error of $19^{\circ}$, which is still sig-

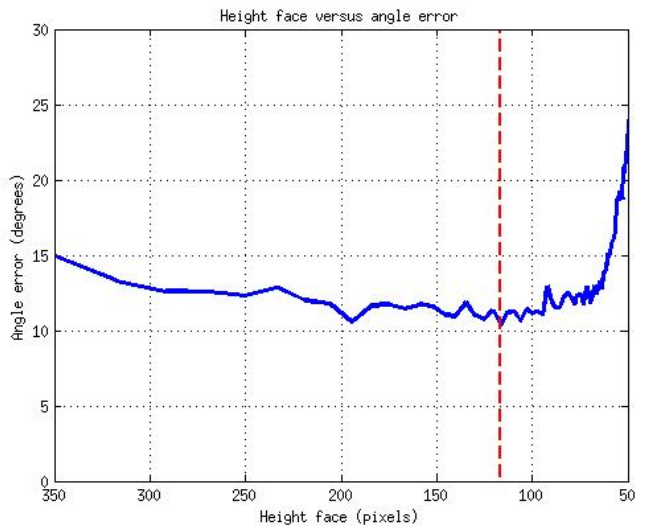

Figure 10: Angular error versus face height. Optimal face height $=117$ pixels which corresponds with a rescale factor of 3 .

nificantly larger than ours and a lot more processing power is needed, which is disadvantageous for embedded applications like ours.

The next experiment we conducted was to observe the effect of image downscaling on the angular error. Downscaling the image yields an acceleration of the algorithm but downscaling too much makes a face undetectable (too small for the V\&J models). Figure 10 illustrates the relation between the height of a face and the angular error of the algorithm. The optimal face-height is 117 pixels. We can also observe that a larger image does not yield a better angular error, this is mainly due to more false detections. Moreover, also for low resolution images with faces of only 75 pixels high, our accuracy is still better than competition.

Next we conducted several speed experiments, both on a normal desktop computer and on the processing platform mounted on our UAV. Our UAV is a Xbird 250 equipped with a Logitech webcam (C310) and a processing board as seen in Figure 11. The processing platform we utilize is an Odroid XU3 credit card sized minicomputer. It contains a Samsung Exynos5422 Cortex-A15 2.0GHz quad core and a Cortex-A7 quad core processor. We optimized the algorithm to run at maximum speed specifically for the Odroid board. In our images, the face height was about $70 \%$ (350 pixels) of the image height (480 pixels). We downscaled the input image with scale factor of 3 which was empirically determined as the most optimal value according to our experiments above (350 pixels / 117 pixels $=3$ ). Most processing time is spent on evaluating the V\&J models, thus downscaling the image results in an acceleration. Both approaches are evaluated on a normal desktop computer and on the Odroid at normal speed (input image is not cropped $($ size $=640 \times 480$ pixels $))$ and at maximum 

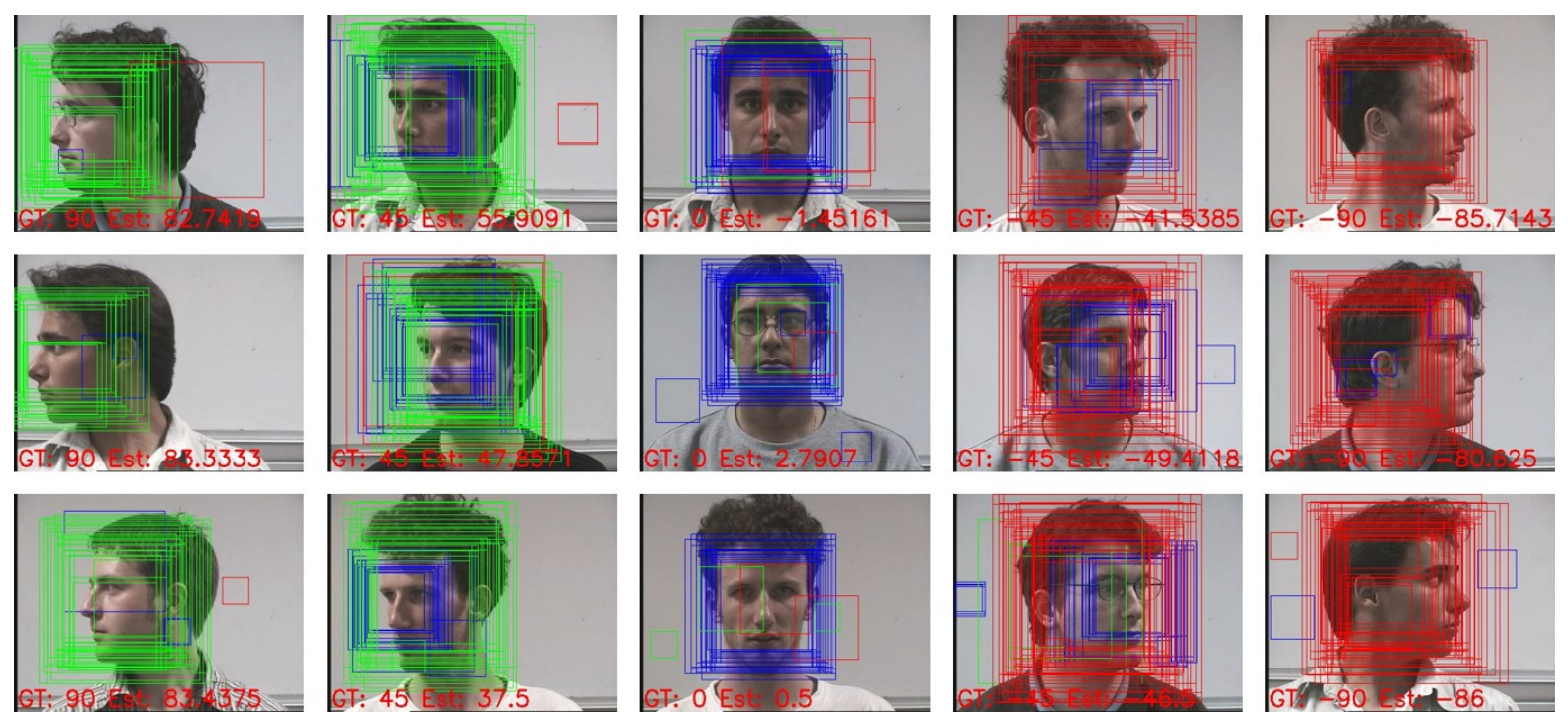

Figure 9: Result of the angle model approach on the dataset of (Gourier et al., 2004). In every image the ground truth angle (GT) and the estimated angle (Est) is displayed. The color of the rectangle correspond with the model that is detected. Green: Left looking model, Blue: Frontal looking model and Red: Right looking model.

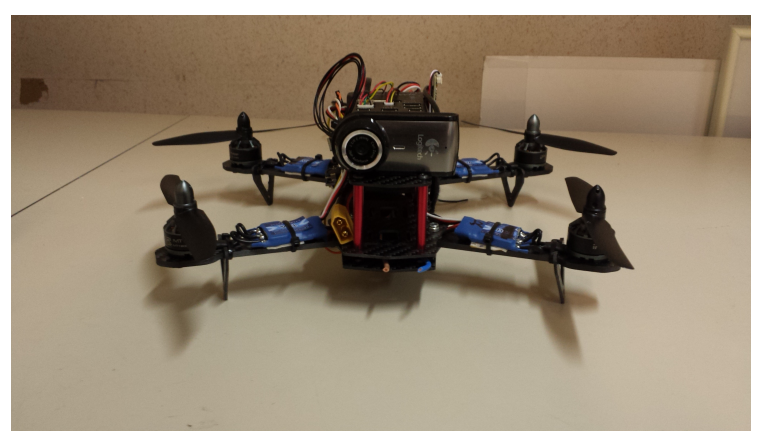

Figure 11: The UAV where we did our experiment with. XBird 250 equipped with an Odroid XU3 processing board.

\begin{tabular}{l|c|c} 
& $\begin{array}{c}\text { Baseline } \\
\text { Approach }\end{array}$ & $\begin{array}{c}\text { Angle Model } \\
\text { Approach }\end{array}$ \\
\hline Desktop avg. (fps) & 13,34 & 13,4 \\
\hline Desktop max (fps) & 73,17 & 73,48 \\
\hline Odroid avg. (fps) & 2,33 & 2,35 \\
\hline Odroid max (fps) & 12,49 & 12,39
\end{tabular}

Table 2: Speed results

speed (image downscaled so that faces are still detectable for our application ( $($ ize $=213 \times 160$ pixels $)$ ).

As seen in Table 2 there is almost no difference in processing speed between the baseline approach and the Angle Model Approach. The image can be downscaled to $213 \times 160$ pixels without loss in accuracy.

We also made a demo towards our UAV application where we used the calculated angle of a face to control a pan-tilt unit with the camera mounted on. The unit turns the camera to position the face more left or right in the image depending on the angle of the face, yielding cinematographically correct mid-shots. A movie of this demo can be seen at https://youtu.be/jprqiRlpap4.

\section{CONCLUSION AND FUTURE WORK}

In this paper we developed two approaches to calculate the pan-angle of a face in an input image between $-90^{\circ}$ and $90^{\circ}$. We used the first approach as a baseline and the second approach as an improvement in accuracy without compromising on processing speed. We validated these two approaches on a publicly available dataset as well as on our own recorded dataset from a UAV. The approaches are also implemented on an Odroid embedded processing platform attached on a UAV which prove that they run in real-time on embedded hardware. Both methods have the advantage that they detect the face and the angle with the same model, which is very beneficial for processing speed. They are not using time consuming decision-trees or facial landmarks but only the relationship between learned angles and their corresponding scores and can work on images with relatively low resolution. Because we made our code, angle model and dataset publicly available ${ }^{1}$ others can implement and use our algorithm easily.

In the future we will integrate this angle estima-

\footnotetext{
${ }^{1}$ www.eavise.be/hulens
} 
tion technology in a virtual cameraman UAV demo, choosing the right position for which to film an actor in order to get a requested shot. Furthermore, we want to test our approaches using LBP features instead of Haar features to train the previous models. Using LBP features would increase the algorithms' processing speed even further (integer calculations instead of float calculations). As seen in Figure 9 false detections occur. Filtering out these false detections will yield in an even better accuracy.

\section{ACKNOWLEDGEMENTS}

This work is supported by KU Leuven via the CAMETRON project.

\section{REFERENCES}

Benfold, B. and Reid, I. (2008). Colour invariant head pose classification in low resolution video. In $B M V C$, pages $1-10$.

Benfold, B. and Reid, I. (2009). Guiding visual surveillance by tracking human attention. In $B M V C$, pages 1-11.

Fanelli, G., Gall, J., and Van Gool, L. (2011). Real time head pose estimation with random regression forests. In Computer Vision and Pattern Recognition (CVPR), 2011 IEEE Conference on, pages 617-624. IEEE.

Fanelli, G., Gall, J., and Van Gool, L. (2012). Real time 3d head pose estimation: Recent achievements and future challenges. In Communications Control and Signal Processing (ISCCSP), 2012 5th International Symposium on, pages 1-4. IEEE.

Gourier, N., Hall, D., and Crowley, J. L. (2004). Estimating face orientation from robust detection of salient facial structures. In FG Net Workshop on Visual Observation of Deictic Gestures, pages 1-9. FGnet (IST2000-26434) Cambridge, UK.

Hulens, D., Goedemé, T., and Rumes, T. (2014). Autonomous lecture recording with a ptz camera while complying with cinematographic rules. In Computer and Robot Vision (CRV), 2014 Canadian Conference on, pages 371-377. IEEE.

Liew, C. F. and Yairi, T. (2015). Human head pose estimation and its application in unmanned aerial vehicle control. In The Malaysia-Japan Model on Technology Partnership, pages 327-336. Springer.

Liu, Y., Wang, Q., Jiang, Y., and Lei, Y. (2014). Supervised locality discriminant manifold learning for head pose estimation. Knowledge-Based Systems, 66:126-135.

Lu, J. and Tan, Y.-P. (2013). Ordinary preserving manifold analysis for human age and head pose estimation. Human-Machine Systems, IEEE Transactions on, 43(2):249-258.

Marks, T. and Jones, M. (2015). Real-time head pose estimation and facial feature localization using a depth sensor and triangular surface patch features.
Oyini Mbouna, R., Kong, S. G., and Chun, M.-G. (2013). Visual analysis of eye state and head pose for driver alertness monitoring. Intelligent Transportation Systems, IEEE Transactions on, 14(3):1462-1469.

Paone, J., Bolme, D., Ferrell, R., Aykac, D., and Karnowski, T. (2015). Baseline face detection, head pose estimation, and coarse direction detection for facial data in the shrp2 naturalistic driving study. In Intelligent Vehicles Symposium (IV), 2015 IEEE, pages 174-179. IEEE.

Pyun, N.-J., Sayah, H., and Vincent, N. (2014). Adaptive haar-like features for head pose estimation. In Image Analysis and Recognition, pages 94-101. Springer.

Rehder, E., Kloeden, H., and Stiller, C. (2014). Head detection and orientation estimation for pedestrian safety. In Intelligent Transportation Systems (ITSC), 2014 IEEE 17th International Conference on, pages 2292 2297. IEEE.

Schulz, A. and Stiefelhagen, R. (2012). Video-based pedestrian head pose estimation for risk assessment. In Intelligent Transportation Systems (ITSC), 2012 15th International IEEE Conference on, pages 1771-1776. IEEE.

Shbib, R., Zhou, S., Ndzi, D., and Alkadhimi, K. (2014). Head pose estimation for car drivers. International Journal of u-and e-Service, Science and Technology, 7(4):359-374.

Tawari, A., Martin, S., and Trivedi, M. M. (2014). Continuous head movement estimator for driver assistance: Issues, algorithms, and on-road evaluations. Intelligent Transportation Systems, IEEE Transactions on, 15(2):818-830.

Viola, P. and Jones, M. (2001). Rapid object detection using a boosted cascade of simple features. In Computer Vision and Pattern Recognition, 2001. CVPR 2001. Proceedings of the 2001 IEEE Computer Society Conference on, volume 1, pages I-511. IEEE.

Yano, S., Gu, Y., and Kamijo, S. (2014). Estimation of pedestrian pose and orientation using on-board camera with histograms of oriented gradients features. International Journal of Intelligent Transportation Systems Research, pages 1-10. 